Proceedings of the Estonian Academy of Sciences,

$2021,70,1,62-79$

https://doi.org/10.3176/proc.2021.1.09

CONTROL

Available online at www.eap.ee/proceedings

THEORY

\title{
Linearization of discrete-time control system by state transformation
}

\author{
Tanel Mullari ${ }^{\mathrm{a} *}$ and Ülle Kotta ${ }^{\mathrm{b}}$ \\ a Tallinn University of Technology, Department of Cybernetics, Ehitajate tee 5, 19086 Tallinn, Estonia \\ b Tallinn University of Technology, Department of Software Sciences, Akadeemia tee 21/1, 12618 Tallinn, Estonia
}

Received 15 October 2020, accepted 10 December 2020, available online 10 February 2021

(C) 2021 Authors. This is an Open Access article distributed under the terms and conditions of the Creative Commons AttributionNonCommercial 4.0 International License (http://creativecommons.org/licenses/by-nc/4.0/).

\begin{abstract}
This paper presents necessary and sufficient linearizability conditions by state transformation for discrete-time multiinput nonlinear control system under the mild assumption on the surjectivity property and describes how to find the state transformation when it exists. The conditions are formulated in terms of backward shifts of vector fields, defined by the system dynamics. The conditions are compared with those that allow additionally the regular static state feedback. The theory is illustrated by two examples.
\end{abstract}

Key words: nonlinear control system, discrete-time system, backward shifts of vector fields, state linearizability.

\section{INTRODUCTION}

Linearization of system dynamics by state transformation (and state feedback), when applicable, is an extremely powerful technique. Once such linearization is obtained, one can rely on the linear control theory to control nonlinear systems. The focus of this paper is on discrete-time nonlinear control systems. In [1] the algebraic framework was developed for such systems, based on the shifts of the vector fields, and being dual to the framework based on the differential forms [2,3]. It is important to note that the new framework does not assume system reversibility like the other approaches to nonlinear discrete-time systems [4,5]; only a less restrictive surjectivity property is necessary. Moreover, in [1] the alterative characterization of the static state feedback linearizability property was given in terms of the vector spaces of the backward shifts of certain vector fields, defined by system dynamics. Let us recall that the static state feedback linearizability property means that by using the state transformation and regular static state feedback, one can transform the state equations into the form such that the forward shifts of the state coordinates are described by the linear functions of the states and inputs. The goal of this paper is to find conditions, again in terms of the backward shifts of certain vector fields, under which the multi-input system can be linearized, using only the state transformation. Since feedback is not allowed, the conditions are obviously more restrictive than those regarding the static state feedback linearizability in [1]. It should be pointed out that the problem was addressed earlier in [4] for single-input systems by using different tools. Moreover, it is important to mention that we look for conditions in the form that include those from [1] plus some additional conditions,

\footnotetext{
* Corresponding author, tanel.mullari@taltech.ee
} 
so it will be clear by direct inspection how much freedom is lost compared with the case where feedback is also used.

Linearization by state transformation for a single-input system is also studied in [6], where it has been proven that the state linearizability property can be alternatively characterized by the specific structure of system dynamics, described in terms of the composition of two diffeomorphisms. It should be noted that such characterization holds only under the assumption of system reversibility.

Finally, unlike in [4], where local results were given, our results are generic, i.e. valid on an open and dense subset of the state space, provided that they hold at some point of the domain. Let us mention that the concept of the generic property does not make sense, in general, for systems described by smooth functions, whereas it makes sense in the case of analytic functions [7]. For this reason we assume analyticity, whereas the framework in $[4,5]$ requires only smoothness. Since we look at dimensions (or ranks) over the field of functions and not over $\mathbb{R}$, it is meaningless to speak about constant dimensionality of the distributions. A generic rank is a maximal rank on an open and dense set. The rank may drop on some subset. By reducing the set, one can always achieve a constant rank over $\mathbb{R}$.

\section{PRELIMINARIES}

Let us recall some preliminary results that originate mostly from [1] and consider the discrete-time nonlinear control system

$$
x^{\langle 1\rangle}(t)=\bar{\Phi}(x(t), u(t))
$$

where $x^{\langle 1\rangle}(t):=x(t+1), t \in \mathbb{Z}$, the variables $x(t) \in \bar{X} \subset \mathbb{R}^{n}, u(t) \in U \subset \mathbb{R}^{m}$, and the state transition map $\bar{\Phi}: \bar{X} \times U \rightarrow \bar{X}$ is supposed to be analytic. Both $\bar{X}$ and $U$ are assumed to be open sets. We assume that the map $\bar{\Phi}$ can be extended to the map $\Phi=\left[\bar{\Phi}^{T}, \chi^{T}\right]^{T}: \bar{X} \times U \rightarrow \bar{X} \times \mathbb{R}^{m}$ so that $\Phi$ has the global analytic inverse $\left[\Lambda^{T}, \lambda^{T}\right]^{T}: \Phi(\bar{X} \times U) \rightarrow \bar{X} \times U$. We introduce the additional variable $z(t) \in \mathbb{R}^{m}$,

$$
z(t)=\chi(x(t), u(t))
$$

The system (1), (2) defines the inversive difference field $\mathscr{K}$ of the meromorphic functions in a finite number of variables from the set $\mathscr{C}=\left\{x, u^{\langle k\rangle}, z^{\langle-l\rangle}, k \geq 0, l \geq 1\right\}$. Here $x^{\langle k\rangle}$ denotes the $k$ th order forward shift of $x$ and $z^{\langle-l\rangle}$ the $l$ th order backward shift of $z$. The 1st order forward shift of the variable $x$ is defined by the equations (1) and the 1st order backward shifts of $x$ and $u$ by

$$
x^{\langle-1\rangle}=\Lambda\left(x, z^{\langle-1\rangle}\right), \quad u^{\langle-1\rangle}=\lambda\left(x, z^{\langle-1\rangle}\right) .
$$

The forward shift of a function $\varphi\left(x, u, u^{\langle 1\rangle}, \ldots, u^{\langle k\rangle}, z^{\langle-1\rangle}, \ldots, z^{\langle-l\rangle}\right) \in \mathscr{K}$ is defined as the composition $\varphi^{\langle 1\rangle}:=\varphi\left(\Phi(x, u), u^{\langle 1\rangle}, u^{\langle 2\rangle}, \ldots, u^{\langle k+1\rangle}, \chi(x, u), \ldots, z^{\langle-l+1\rangle}\right)$. The higher order forward shifts of $x$ are defined recursively as

$$
\begin{aligned}
x^{\langle 1\rangle} & =\bar{\Phi}^{1}(x, u):=\bar{\Phi}(x, u), \\
x^{\langle k\rangle} & =\bar{\Phi}^{k}\left(x, u, u^{\langle 1\rangle}, \ldots, u^{\langle k-1\rangle}\right):=\bar{\Phi}^{\langle k-1\rangle}\left(\bar{\Phi}(x, u), u^{\langle 1\rangle}, \ldots, u^{\langle k-1\rangle}\right), \quad k \geq 2 .
\end{aligned}
$$

The backward shift of $\varphi$ is the composition $\varphi^{\langle-1\rangle}:=\varphi\left(\Lambda\left(x, z^{\langle-1\rangle}\right), \lambda\left(x, z^{\langle-1\rangle}\right), u, \ldots, u^{\langle k-1\rangle}, z^{\langle-2\rangle}, \ldots, z^{\langle-l-1\rangle}\right)$. The higher order backward shifts of $x$ are defined recursively as

$$
\begin{aligned}
& x^{\langle-1\rangle}=\Lambda^{1}\left(x, z^{\langle-1\rangle}\right):=\Lambda\left(x, z^{\langle-1\rangle}\right), \\
& x^{\langle-k\rangle}=\Lambda^{k}\left(x, z^{\langle-1\rangle}, z^{\langle-2\rangle}, \ldots, z^{\langle-k\rangle}\right):=\Lambda^{k-1}\left(\Lambda\left(x, z^{\langle-1\rangle}\right), z^{\langle-2\rangle}, \ldots, z^{\langle-k\rangle}\right), \quad k \geq 2 .
\end{aligned}
$$


Due to (2) and (4), the higher order forward shifts of $z$ can be computed as $z^{\langle k\rangle}=\chi\left(\bar{\Phi}^{k}\left(x, u, \ldots, u^{\langle k-1\rangle}\right), u^{\langle k\rangle}\right)$, and due to (3) and (5), the higher order backward shifts of $u$ as $u^{\langle-k\rangle}=\lambda\left(\Lambda^{k-1}\left(x, z^{\langle-1\rangle}, \ldots, z^{\langle-k+1\rangle}\right), z^{\langle-k\rangle}\right)$. Consider the infinite set of the symbols $\mathrm{d} \mathscr{C}=\left\{\mathrm{d} x, \mathrm{~d} u^{\langle k\rangle}, \mathrm{d} z^{\langle-l\rangle}, k \geq 0, l \geq 1\right\}$ and let $\mathscr{E}:=\operatorname{span}_{\mathscr{K}}\{\mathrm{d} \mathscr{C}\}$ be the vector space spanned over $\mathscr{K}$ by the elements of $\mathrm{d} \mathscr{C}$, called the 1 -forms

$$
\omega=\sum_{i=1}^{n} A_{i} \mathrm{~d} x_{i}+\sum_{k \geq 0} \sum_{j=1}^{m} B_{j k} \mathrm{~d} u_{j}^{\langle k\rangle}+\sum_{l \geq 1} \sum_{q=1}^{m} C_{q l} \mathrm{~d} z_{q}^{\langle-l\rangle},
$$

where only the finite number of $B_{j k}$ and $C_{q l}$ differ from zero [2]. Next define the space $\mathscr{E}^{*}$, dual to $\mathscr{E}$, whose elements are the vector fields

$$
\Xi=\sum_{i=1}^{n} \xi_{i} \frac{\partial}{\partial x_{i}}+\sum_{k \geq 0} \sum_{j=1}^{m} \xi_{j k} \frac{\partial}{\partial u_{j}^{\langle k\rangle}}+\sum_{l \geq 1} \sum_{q=1}^{m} \bar{\xi}_{q l} \frac{\partial}{\partial z_{q}^{\langle-l\rangle}} .
$$

By duality between $\mathscr{E}$ and $\mathscr{E}^{*}$ the following holds for scalar products of 1-forms and the vector fields: $\left\langle\mathrm{d} x_{i}, \Xi\right\rangle=\xi_{i},\left\langle\mathrm{~d} u_{j}^{\langle k\rangle}, \Xi\right\rangle=\xi_{j k},\left\langle\mathrm{~d} z_{q}^{\langle-l\rangle}, \Xi\right\rangle=\xi_{q l}$.

The backward shift of the vector field $\Xi$ in (6) is another vector field

$$
\Xi^{\langle-1\rangle}=\sum_{i=1}^{n} a_{i} \frac{\partial}{\partial x_{i}}+\sum_{k \geq 0} \sum_{j=1}^{m} b_{j k} \frac{\partial}{\partial u_{j}^{\langle k\rangle}}+\sum_{l \geq 1} \sum_{q=1}^{m} c_{q l} \frac{\partial}{\partial z_{q}^{\langle-l\rangle}}
$$

where

$$
a_{i}=\left\langle\mathrm{d} x_{i}^{\langle 1\rangle}, \Xi\right\rangle^{\langle-1\rangle}=\left\langle\mathrm{d} \bar{\Phi}_{i}, \Xi\right\rangle^{\langle-1\rangle}, b_{j k}=\left\langle\mathrm{d} u_{j}^{\langle k+1\rangle}, \Xi\right\rangle^{\langle-1\rangle}, c_{q l}=\left\langle\mathrm{d} z_{q}^{\langle-l+1\rangle}, \Xi\right\rangle^{\langle-1\rangle} .
$$

The projection of $\Xi$ in (6) is another vector field

$$
\Xi^{\pi}=\sum_{i=1}^{n} \xi_{i} \frac{\partial}{\partial x_{i}}
$$

Note that the shift and the projection operators do not commute. For simplicity of notation, in what follows, we often write

$$
\partial / \partial x=\left(\partial / \partial x_{1}, \ldots, \partial / \partial x_{n}\right)^{T}, \partial / \partial u=\left(\partial / \partial u_{1}, \ldots, \partial / \partial u_{m}\right)^{T}, \partial / \partial z^{\langle-l\rangle}=\left(\partial / \partial z_{1}^{\langle-l\rangle}, \ldots, \partial / \partial z_{m}^{\langle-l\rangle}\right)^{T} .
$$

Recall that the static state feedback linearizability conditions for system (1) are formulated in terms of a non-decreasing sequence of the distributions of vector fields, defined for $k \geq 1$ by

$$
\mathscr{D}_{k}=\operatorname{span}_{\mathscr{K}}\left\{\frac{\partial}{\partial z^{\langle-1\rangle}},\left(\frac{\partial}{\partial u}\right)^{\langle-l\rangle \pi}, \quad l=1, \ldots, k\right\} .
$$

Like in [1], $k^{*}$ denotes a uniquely defined integer such that $\operatorname{dim}_{\mathscr{K}} \mathscr{D}_{k}<\operatorname{dim}_{\mathscr{K}} \mathscr{D}_{k^{\star}}$ for $k<k^{\star}$, but $\operatorname{dim}_{\mathscr{K}} \mathscr{D}_{k}=$ $\operatorname{dim}_{\mathscr{K}} \mathscr{D}_{k^{\star}}$ for all $k \geq k^{\star}$. Define also the projections $\mathscr{D}_{k}^{\pi}$ as

$$
\mathscr{D}_{k}^{\pi}=\mathscr{D}_{k} \cap \operatorname{span}_{\mathscr{K}}\left\{\frac{\partial}{\partial x}\right\}=\operatorname{span}_{\mathscr{K}}\left\{\left(\frac{\partial}{\partial u}\right)^{\langle-l\rangle \pi}, \quad l=1, \ldots, k\right\} .
$$

Lemma 1. [1] System (1) is accessible if and only if $\operatorname{dim}_{\mathscr{K}} \mathscr{D}_{k^{\star}}^{\pi}=n$. 
Remark 2. The fact that $\operatorname{dim}_{\mathscr{K}} \mathscr{D}_{k^{\star}}^{\pi}=n$ implies that there exist $m$ indices $n_{i}, n_{i} \geq 1, \sum_{i=1}^{m} n_{i}=n$, such that

$$
\operatorname{dim}_{\mathscr{K}} \operatorname{span}_{\mathscr{K}}\left\{\left(\frac{\partial}{\partial u_{i}}\right)^{\left\langle-l_{i}\right\rangle \pi}, \quad l_{i}=1, \ldots, n_{i}\right\}=n .
$$

Obviously, $k^{\star}=\max \left\{n_{i}\right\}$.

A distibution $\mathscr{D}$ is called involutive, if for the arbitrary vector fields $\Xi_{1}, \Xi_{2} \in \mathscr{D}$ also their Lie brackets $\left[\Xi_{1}, \Xi_{2}\right] \in \mathscr{D}$. The vector fields $\Xi_{1}$ and $\Xi_{2}$ commute, if their Lie brackets are identically equal to zero. It is easy to check by simple calculations that:

$$
\left[\Xi_{1}, \Xi_{2}\right]^{\langle-1\rangle}=\left[\Xi_{1}^{\langle-1\rangle}, \Xi_{2}^{\langle-1\rangle}\right],\left\langle\omega, \Xi_{1}\right\rangle^{\langle-1\rangle}=\left\langle\omega^{\langle-1\rangle}, \Xi_{1}^{\langle-1\rangle}\right\rangle,\left\langle\omega, \Xi_{1}\right\rangle^{\langle 1\rangle}=\left\langle\omega^{\langle 1\rangle}, \Xi_{1}^{\langle 1\rangle}\right\rangle,
$$

where $\left\langle\omega, \Xi_{1}\right\rangle$ denotes the scalar product of a 1-form $\omega$ and a vector field $\Xi_{1}$. For a 1-form $\omega=\sum_{i} A_{i} \mathrm{~d} \zeta_{i}, A_{i} \in$ $\mathscr{K}, \mathrm{d} \zeta_{i} \in \mathrm{d} \mathscr{C}$, the forward and backward shifts are defined as $\omega^{\langle 1\rangle}=\sum_{i} A_{i}^{\langle 1\rangle} \mathrm{d} \zeta_{i}^{\langle 1\rangle}$ and $\omega^{\langle-1\rangle}=\sum_{i} A_{i}^{\langle-1\rangle} \mathrm{d} \zeta_{i}^{\langle-1\rangle}$, respectively.

Consider a vector field $\Xi=\sum_{i=1}^{n} \xi_{i}(x) \partial / \partial x_{i}$, whose coefficients depend only on $x$. Under the state transformation

$$
X=\Psi(x)
$$

this vector field takes, in the new coordinates, the form [8]

$$
\Psi_{*} \Xi=\left.\sum_{i, j=1}^{n} \frac{\partial \Psi_{j}(x)}{\partial x_{i}} \xi_{j}(x)\right|_{x=\Psi^{-1}(X)} \frac{\partial}{\partial X_{j}} .
$$

Here the operator $\Psi_{*}$ is called the tangent map of $\Psi$, and the vector field $\Psi_{*} \Xi$ is called the image of the vector field $\Xi$ under the map $\Psi_{*}$.

A set of vector fields $\Xi_{1}, \ldots, \Xi_{n}$ is called linearly independent if there do not exist non-zero coefficients $\beta_{i} \in \mathscr{K}, i=1, \ldots, n$, such that $\sum_{i=1}^{n} \beta_{i} \Xi_{i} \equiv 0$.

Lemma 3. Let $\Xi_{j}=\sum_{i=1}^{n} \xi_{i j}(x) \partial / \partial x_{i} \in \operatorname{span}_{\mathscr{K}}\{\partial / \partial x\}, j=1, \ldots, n$, be linearly independent. If the vector fields $\Xi_{j}$ commute, then generically one can define the state transformation $X_{i}=\Psi_{i}(x), \Psi_{i} \in \mathscr{K}, i=1, \ldots, n$, such that

$$
\left\langle\mathrm{d} \Psi_{i}, \Xi_{j}\right\rangle=\delta_{i j}, \quad i, j=1, \ldots, n .
$$

Moreover, in the new coordinates

$$
\Psi_{*} \Xi_{j}=\frac{\partial}{\partial X_{j}}
$$

Proof. See Appendix A.

This lemma is an analogue of Theorem 2.36 from [8], which proves the local validity of the result around a fixed point on a manifold. However, the proof is not easy to extend for the generic case, since it relies on shifting the point under the flows of the vector fields $\Xi_{j}, j=1, \ldots, n$. The coordinate transformation can, under commutativity assumption, be constructed as the composition of these flows. Moreover, the approach in this paper is algebraic, not differential geometric. For the above reasons, the alternative proof is given.

The problem statement. System (1) is said to be (generically) linearizable by state transformation (briefly, state linearizable), if there exists an analytic state transformation $X=\Psi(x), \Psi_{i} \in \mathscr{K}, i=1, \ldots, n$, such that the system in the new coordinates takes the form

$$
X^{\langle 1\rangle}=A X+B u,
$$

where the pair $(A, B)$ satisfies the Kalman controllability condition. The main goal of the paper is to derive the necessary and sufficient conditions for the existence of the state transformation (14) that allows to transform the equations (1) into the form (18). 


\section{BACKWARD SHIFTS OF THE VECTOR FIELDS}

In this section we prove several properties of the backward shifts of the vector fields $\partial / \partial u_{i}, i=1, \ldots, m$, necessary for proving the main result. Note that, in general, the vector fields $\Xi$ defined by (6) belong to the infinite dimensional space $\mathscr{E}^{*}$. However, in this paper we operate with the vector fields $\partial / \partial u_{i}, i=1, \ldots, m$, and their backward shifts up to order $k^{\star}+1$. Below we will show that such vector fields belong to the finite space ${\mathscr{E}^{*}}^{*} \operatorname{span}_{\mathscr{K}}\left\{\partial / \partial x, \partial / \partial u, \partial / \partial z^{\langle-l\rangle}, l=1, \ldots, k^{\star}+1\right\}$. Although the backward shifts of $\partial / \partial u_{i}$ for $l \leq k^{\star}+1$, used in the following theorems, belong to the lower dimensional subspaces of $\overline{\mathscr{E}}^{*}$, we consider them as the elements of $\overline{\mathscr{E}}^{*}$.

Lemma 4. The backward shifts of the vector field $\partial / \partial u_{i}$ have, for $l=1, \ldots, k^{\star}+1$, the following properties:

(i)

$$
\left(\frac{\partial}{\partial u_{i}}\right)^{\langle-l\rangle} \in \operatorname{span}_{\mathscr{K}}\left\{\frac{\partial}{\partial x}, \frac{\partial}{\partial z^{\langle-1\rangle}}, \ldots \frac{\partial}{\partial z^{\langle-l\rangle}}\right\},
$$

(ii) their coefficients depend only on the variables $x, z^{\langle-1\rangle}, \ldots, z^{\langle-l\rangle}$.

Proof. See Appendix B.

Lemma 5. For $i, j=1, \ldots, m$ and $l, q=1, \ldots, k^{\star}+1$,

$$
\left[\left(\frac{\partial}{\partial u_{i}}\right)^{\langle-l\rangle},\left(\frac{\partial}{\partial u_{j}}\right)^{\langle-q\rangle}\right] \equiv 0 .
$$

Proof. The proof is by induction. Since the partial derivative operators always commute, (19) holds for $l, q=0$. Suppose that (19) is valid for $l, q=0, \ldots, k$, and let us prove its validity for $k+1$. First, shift (19) backwards by one step, using (13)

$$
\left[\left(\frac{\partial}{\partial u_{i}}\right)^{\langle-l-1\rangle},\left(\frac{\partial}{\partial u_{j}}\right)^{\langle-q-1\rangle}\right] \equiv 0, \quad l, q=0, \ldots, k .
$$

Using notations $\bar{l}:=l+1, \bar{q}:=q+1$ gives

$$
\left[\left(\frac{\partial}{\partial u_{i}}\right)^{\langle-\bar{l}\rangle},\left(\frac{\partial}{\partial u_{j}}\right)^{\langle-\bar{q}\rangle}\right] \equiv 0, \quad \bar{l}, \bar{q}=1, \ldots, k+1 .
$$

From Lemma 4, the coefficients of $\left(\partial / \partial u_{i}\right)^{\langle-l\rangle}, l=1, \ldots, k^{\star}+1$, do not depend on $u$, and therefore

$$
\left[\left(\frac{\partial}{\partial u_{i}}\right)^{\langle-\bar{l}\rangle}, \frac{\partial}{\partial u_{j}}\right] \equiv 0, \quad \bar{l}=1, \ldots, k+1 .
$$

From (20) and (21) taking into account also the commutativity of all $\partial / \partial u_{i}, i=1, \ldots, m$, we get

$$
\left[\left(\frac{\partial}{\partial u_{i}}\right)^{\langle-\bar{l}\rangle},\left(\frac{\partial}{\partial u_{j}}\right)^{\langle-\bar{q}\rangle}\right] \equiv 0, \quad \bar{l}, \bar{q}=0, \ldots, k+1 .
$$


Observe that due to Lemma 4,

$$
\left(\frac{\partial}{\partial u_{i}}\right)^{\langle-l\rangle}=a_{i l}\left(x, \mathbf{z}_{-l}\right) \frac{\partial}{\partial x}+c_{i l}\left(x, \mathbf{z}_{-l}\right) \frac{\partial}{\partial \mathbf{z}_{-l}}, \quad l=1, \ldots, k^{\star}+1,
$$

where $\mathbf{z}_{-l}:=\left\{z^{\langle-1\rangle}, \ldots, z^{\langle-l\rangle}\right\}$. The projections of $\left(\partial / \partial u_{i}\right)^{\langle-l\rangle}$ read, according to (9) as

$$
\left(\frac{\partial}{\partial u_{i}}\right)^{\langle-l\rangle \pi}=a_{i l}\left(x, \mathbf{z}_{-l}\right) \frac{\partial}{\partial x}
$$

Lemma 6. If the coefficients of $\left(\partial / \partial u_{i}\right)^{\langle-l\rangle \pi}$ and the coefficients of $\left(\partial / \partial u_{j}\right)^{\langle-q\rangle}$, $i, j=1, \ldots, m$ and $l, q=1, \ldots, k^{\star}+1$, depend only on $x$, then

$$
\left[\left(\frac{\partial}{\partial u_{i}}\right)^{\langle-l\rangle \pi},\left(\frac{\partial}{\partial u_{j}}\right)^{\langle-q\rangle \pi}\right] \equiv 0 .
$$

Proof. According to (22) and the definition of the Lie brackets

$$
\begin{aligned}
& {\left[\left(\frac{\partial}{\partial u_{i}}\right)^{\langle-l\rangle},\left(\frac{\partial}{\partial u_{j}}\right)^{\langle-q\rangle}\right]} \\
& =\left(\frac{\partial a_{j q}}{\partial x} a_{i l}-\frac{\partial a_{i l}}{\partial x} a_{j q}+\frac{\partial a_{j q}}{\partial \mathbf{z}_{-p}} c_{i l}-\frac{\partial a_{i l}}{\partial \mathbf{z}_{-p}} c_{j q}\right) \frac{\partial}{\partial x}+\left(\frac{\partial c_{j q}}{\partial x} a_{i l}-\frac{\partial c_{i l}}{\partial x} a_{j q}+\frac{\partial c_{j q}}{\partial \mathbf{z}_{-p}} c_{i l}-\frac{\partial c_{i l}}{\partial \mathbf{z}_{-p}} c_{j q}\right) \frac{\partial}{\partial \mathbf{z}_{-p}}
\end{aligned}
$$

where $p=\max \{l, q\}$. Due to Lemma 5, the left hand side of the above equality is identically equal to zero. Therefore, both expressions in the parentheses on the right hand side of the equality must be also identically equal to zero and thus:

$$
\frac{\partial a_{j q}}{\partial x} a_{i l}-\frac{\partial a_{i l}}{\partial x} a_{j q}+\frac{\partial a_{j q}}{\partial \mathbf{z}_{-p}} c_{i l}-\frac{\partial a_{i l}}{\partial \mathbf{z}_{-p}} c_{j q} \equiv 0
$$

By the assumption of the lemma, the quantities $a_{i l}$ and $a_{j q}$ as the coefficients of the vector fields $\left(\partial / \partial u_{i}\right)^{\langle-l\rangle}$ and $\left(\partial / \partial u_{j}\right)^{\langle-q\rangle}$, respectively, depend only on $x$, and so the equality (25) takes the form

$$
\frac{\partial a_{j q}}{\partial x} a_{i l}-\frac{\partial a_{i l}}{\partial x} a_{j q} \equiv 0
$$

According to (23) and the definition of the Lie bracket, the quantities on the left hand side of (26) are the coefficients of the Lie brackets in (24).

Lemma 7. If the coefficients of $\left(\partial / \partial u_{i}\right)^{\left\langle-l_{i}\right\rangle \pi}, i=1, \ldots, m, l_{i}=1, \ldots, n_{i}+1$, do not depend on $z^{\langle-1\rangle}$, then they are the functions of $x$ only.

Proof. According to Lemma 4 the coefficients of $\left(\partial / \partial u_{i}\right)^{\left\langle-l_{i}\right\rangle \pi}$ depend on the variables $x, z^{\langle-1\rangle}, \ldots, z^{\left\langle-l_{i}\right\rangle}$ only, see (23). Consequently, we have to show that if these coefficients do not depend on $z^{\langle-1\rangle}$, then they do not depend on the higher order backward shifts of $z$ either. The proof is by induction. Due to Lemma 4 the coefficients of $\left(\partial / \partial u_{i}\right)^{\langle-1\rangle}$ are the functions of $x$ and $z^{\langle-1\rangle}$ only, i.e. in case $l_{i}=1$, the lemma holds.

Assume now that $l_{i}=k$. Suppose that the coefficients of $\left(\partial / \partial u_{i}\right)^{\langle-k\rangle}$ depend only on $x$, i.e.

$$
\left(\frac{\partial}{\partial u_{i}}\right)^{\langle-k\rangle \pi}=\sum_{j=1}^{n} a_{j i k}(x) \frac{\partial}{\partial x_{j}} .
$$


Show that in this case the coefficients of $\left(\partial / \partial u_{i}\right)^{\langle-k-1\rangle \pi}$ depend only on $x$ and $z^{\langle-1\rangle}$. According to (7), (8) and (9),

$$
\left(\frac{\partial}{\partial u_{i}}\right)^{\langle-k-1\rangle \pi}=\sum_{p=1}^{n}\left\langle\mathrm{~d} \bar{\Phi}_{p},\left(\frac{\partial}{\partial u_{i}}\right)^{\langle-k\rangle \pi}\right\rangle^{\langle-1\rangle} \frac{\partial}{\partial x_{p}}=\sum_{j, p=1}^{n}\left(\frac{\partial \bar{\Phi}}{\partial x_{j}} a_{j i k}\right)^{\langle-1\rangle} \frac{\partial}{\partial x_{p}} .
$$

Note that the product in the parentheses above depends only on $x$ and $u$. Therefore, due to (3), its backward shift depends only on $x$ and $z^{\langle-1\rangle}$. It means that if the coefficients of $\left(\partial / \partial u_{i}\right)^{\langle-k-1\rangle}$ do not depend on $z^{\langle-1\rangle}$, they cannot depend on the higher order backward shifts of $z$ either, i.e. they are the functions of $x$ only.

Corollary 8. If the coefficients of $\left(\partial / \partial u_{i}\right)^{\langle-l\rangle \pi}$ and $\left(\partial / \partial u_{j}\right)^{\langle-q\rangle}, i, j=1, \ldots, m, l=1, \ldots, n_{i}, q=1, \ldots, n_{j}$, do not depend on $z^{\langle-1\rangle}$, then (24) holds.

Proof. Follows directly from Lemma 6 and Lemma 7.

\section{STATE LINEARIZABILITY}

We formulate now the main result of the paper.

Theorem 9. System (1) is generically state linearizable if and only if

(a) there exist $n$ vector fields $\left(\partial / \partial u_{i}\right)^{\left\langle-l_{i}\right\rangle}, i=1, \ldots, m, l_{i}=1, \ldots, n_{i}, \sum_{i=1}^{m} n_{i}=n$, such that (12) holds,

(b) the coefficients of the vector fields $\left(\partial / \partial u_{i}\right)^{\left\langle-l_{i}\right\rangle \pi}, l_{i}=1, \ldots, n_{i}+1$, depend only on the variable $x$.

Proof. Necessity. Suppose that (1) is state linearizable and compute from (18), taking into account that $A^{0}=I$,

$$
\left(\frac{\partial}{\partial u}\right)^{\langle-l\rangle \pi}=A^{l-1} B \frac{\partial}{\partial X}, \quad l=1, \ldots, k^{\star}+1,
$$

where $k^{\star}=\max \left\{n_{i}\right\}$. For such vector fields the condition (b) obviously holds. According to Lemma 1 and Remark 2, the condition (a) is the generic accessibility condition and since the linearized system (18) satisfies the Kalman controllability condition, i.e is accessible, the condition (a) has to be satisfied for system (1) since the state transformation does not change the accessibility property of the system.

Sufficiency. In the sufficiency part of the proof we assume that $i, j=1, \ldots, m$. We first construct, under the conditions (a) and (b), a state transformation and then show that in the new coordinates the system equations are linear. Then, from condition (a) follows that the linear system satisfies the Kalman controllability condition.

Due to Lemma 4 and Corollary 8 , the condition (b) is equivalent to

$$
\left[\frac{\partial}{\partial z^{\langle-1\rangle}},\left(\frac{\partial}{\partial u_{i}}\right)^{\left\langle-l_{i}\right\rangle \pi}\right] \equiv 0, \quad l_{i}=1, \ldots, n_{i}+1 .
$$

Now, by Lemma 6 , for $l_{i}=1, \ldots, n_{i}+1, l_{j}=1, \ldots, n_{j}+1$,

$$
\left[\left(\frac{\partial}{\partial u_{i}}\right)^{\left\langle-l_{i}\right\rangle \pi},\left(\frac{\partial}{\partial u_{j}}\right)^{\left\langle-l_{j}\right\rangle \pi}\right] \equiv 0 .
$$


By (12) and Lemma 4, the projections of the backward shifts of $\partial / \partial u_{i}$ up to order $n_{i}$ build the (commutative) basis for $\operatorname{span}_{\mathscr{K}}\{\partial / \partial x\}$, i.e.

$$
\operatorname{span}_{\mathscr{K}}\left\{\left(\frac{\partial}{\partial u_{i}}\right)^{\left\langle-l_{i}\right\rangle \pi}, i=1, \ldots, m, l_{i}=1, \ldots, n_{i}\right\}=\operatorname{span}_{\mathscr{K}}\left\{\frac{\partial}{\partial x}\right\},
$$

and so the projections of the higher order backward shifts of $\partial / \partial u_{i}$ are their linear combinations

$$
\left(\frac{\partial}{\partial u_{i}}\right)^{\left\langle-n_{i}-1\right\rangle \pi}=\sum_{j=1}^{m} \sum_{l_{j}=1}^{n_{j}} \tilde{a}_{i j l_{j}}(x)\left(\frac{\partial}{\partial u_{j}}\right)^{\left\langle-l_{j}\right\rangle \pi} .
$$

We next prove that all coefficients $\tilde{a}_{i j l_{j}}(x)$ are constants. Note that, due to (28), the coefficients $\tilde{a}_{i j l_{j}}$ do not depend on $\mathbf{z}$. Let us show that they do not depend on $x$ either. From (29) it is immediate that the vector field (31) must commute with all $\left(\partial / \partial u_{k}\right)^{\left\langle-l_{k}\right\rangle \pi}, k=1, \ldots, m, l_{k}=1, \ldots, n_{k}$, i.e.

$$
\sum_{j=1}^{m} \sum_{l_{j}=1}^{n_{j}}\left(\left\langle\mathrm{~d} \tilde{a}_{i j l_{j}},\left(\frac{\partial}{\partial u_{k}}\right)^{\left\langle-l_{k}\right\rangle \pi}\right\rangle\left(\frac{\partial}{\partial u_{j}}\right)^{\left\langle-l_{j}\right\rangle \pi}+\tilde{a}_{i j l_{j}}\left[\left(\frac{\partial}{\partial u_{k}}\right)^{\left\langle-l_{k}\right\rangle \pi},\left(\frac{\partial}{\partial u_{k}}\right)^{\left\langle-n_{k}\right\rangle \pi}\right]\right) \equiv 0 .
$$

By (29), the above equality holds only if for $k=1, \ldots, m, l_{k}=1, \ldots, n_{k}$,

$$
\left\langle\mathrm{d} \tilde{a}_{i j l_{j}},\left(\frac{\partial}{\partial u_{k}}\right)^{\left\langle-l_{k}\right\rangle \pi}\right\rangle \equiv 0
$$

Given (30), the vector fields $\partial / \partial x_{q}, q=1, \ldots, n$, can be written as linear combinations of $\left(\partial / \partial u_{i}\right)^{\left\langle-l_{i}\right\rangle \pi}, i=1, \ldots, m, l_{i}=1, \ldots, n_{i}$. Then from (32) really follows that $\partial \tilde{a}_{i j l_{j}} / \partial x_{q} \equiv 0$. Consequently, all $\tilde{a}_{i j l_{j}}$ are constants.

We will construct now the state transformation $X=\Psi(x)$. Since (29) and (30) hold, then according to Lemma 3, one can define a coordinate transformation $X_{i, n_{i}-l_{i}+1}=\Psi_{i, n_{i}-l_{i}+1}(x), l_{i}=1, \ldots, n_{i}$, such that

$$
\left\langle\mathrm{d} \Psi_{i, n_{i}-l_{i}+1},\left(\frac{\partial}{\partial u_{j}}\right)^{\left\langle-l_{j}\right\rangle \pi}\right\rangle \equiv \delta_{i j} \delta_{l_{i} l_{j}}
$$

where the vector fields $\left(\partial / \partial u_{j}\right)\left\langle-l_{j}\right\rangle \pi, l_{j}=1, \ldots, n_{j}$, will be transformed into the partial derivative operators

$$
\Psi_{*}\left(\frac{\partial}{\partial u_{j}}\right)^{\left\langle-l_{j}\right\rangle \pi}=\frac{\partial}{\partial X_{j, n_{j}-l_{j}+1}} .
$$

Note that here we define the components of the coordinate transformation $\Psi(x)$ (and the new coordinates $X$ ) by using two indices, unlike in Lemma 3 . The reason is that $\Psi_{i, n_{i}-l_{i}+1}, i=1, \ldots, m, l_{i}=1, \ldots, n_{i}$, in (33) are defined as the canonical parameters of the vector fields $\left(\partial / \partial u_{i}\right)^{\left\langle-l_{i}\right\rangle \pi}$. The first index $i$ points to the component of the input $u$, i.e. $u_{i}$, and the second index $n_{i}-l_{i}+1$ refers to the canonical parameter of the projection of the $l_{i}$-th backward shift of $\partial / \partial u_{i}$.

It remains to prove that in the new coordinates the state equations have the form (18), meaning that the forward shift of the vector variable $X$ is the linear vector function of the variables $X$ and $u$. The task is, therefore, to show that for $l_{i}=1, \ldots, n_{i}, l_{j}=1, \ldots, n_{j}$,

$$
\left\langle\mathrm{d} X_{i, n_{i}-l_{i}+1}^{\langle 1\rangle}, \frac{\partial}{\partial X_{j, n_{j}-l_{j}+1}}\right\rangle \equiv \mathrm{const},\left\langle\mathrm{d} X_{i, n_{i}-l_{i}+1}^{\langle 1\rangle}, \frac{\partial}{\partial u_{j}}\right\rangle \equiv \text { const. }
$$


Since the constants are invariant under the coordinate transformations, the identities (35) are likewise valid in the original coordinates and, taking into account also (34), we get, for $l_{i}=1, \ldots, n_{i}, l_{j}=1, \ldots, n_{j}$, the system of equations

$$
\left\langle\mathrm{d} \Psi_{i, n_{i}-l_{i}+1}^{\langle 1\rangle},\left(\frac{\partial}{\partial u_{j}}\right)^{\left\langle-l_{j}\right\rangle \pi}\right\rangle \equiv \mathrm{const},\left\langle\mathrm{d} \Psi_{i, n_{i}-l_{i}+1}^{\langle 1\rangle}, \frac{\partial}{\partial u_{j}}\right\rangle \equiv \text { const. }
$$

In the first equality of (36) one can replace the projections of $\left(\partial / \partial u_{j}\right)^{\left\langle-l_{j}\right\rangle}$ by the vector fields themselves, since the functions $\Psi_{i, n_{i}-l_{i}+1}^{\langle 1\rangle}$ depend only on $x$. This allows (36) to be rewritten, for $l_{i}=1, \ldots, n_{i}, l_{j}=0, \ldots, n_{j}$, as a single equality

$$
\left\langle\mathrm{d} \Psi_{i, n_{i}-l_{i}+1}^{\langle 1\rangle},\left(\frac{\partial}{\partial u_{j}}\right)^{\left\langle-l_{j}\right\rangle}\right\rangle \equiv \text { const. }
$$

In what follows we will show the validity of (37). Let us prove it first for $l_{i}=1, \ldots, n_{i}$ and $l_{j}=0, \ldots, n_{j}-1$. Since all $\Psi_{i, n_{i}-l_{i}+1}$ depend only on $x$, one can replace in (33) $\left(\partial / \partial u_{j}\right)\left\langle-l_{j}\right\rangle \pi$ by $\left(\partial / \partial u_{j}\right)^{\left\langle-l_{j}\right\rangle}$. Shifting (33) forward and taking the above observation into account gives, for $l_{i}=1, \ldots, n_{i}, l_{j}=1, \ldots, n_{j}$,

$$
\left\langle\mathrm{d} \Psi_{i, n_{i}-l_{i}+1}^{\langle 1\rangle},\left(\frac{\partial}{\partial u_{j}}\right)^{\left\langle-l_{j}+1\right\rangle}\right\rangle \equiv \delta_{i j} \delta_{l_{i} l_{j}} \text {. }
$$

If we use the notation $\bar{l}_{j}:=l_{i}-1$ in (38), it is obvious that (37) holds for the values of $l_{j}=0, \ldots, n_{j}-1$. Next prove the validity of (37) for $l_{j}=n_{j}$. From (31), (33) and the fact that $\Psi_{i, n_{i}-l_{i}+1}$ depends only on the variable $x$, one gets

$$
\left\langle\mathrm{d} \Psi_{i, n_{i}-l_{i}+1},\left(\frac{\partial}{\partial u_{j}}\right)^{\left\langle-n_{j}-1\right\rangle}\right\rangle=\sum_{q=1}^{m} \sum_{l_{q}=1}^{n_{q}} \tilde{a}_{j q l_{q}}\left\langle\mathrm{~d} \Psi_{i, n_{i}-l_{i}+1},\left(\frac{\partial}{\partial u_{q}}\right)^{\left\langle-l_{q}\right\rangle \pi}\right\rangle \equiv \tilde{a}_{j i l_{i}} .
$$

Recall that $\tilde{a}_{j i l_{i}}$ is constant. Shifting next (39) forward by one step and taking into account that the forward shift operator does not change a constant, we get

$$
\left\langle\mathrm{d} \Psi_{i, n_{i}-l_{i}+1}^{\langle 1\rangle},\left(\frac{\partial}{\partial u_{j}}\right)^{\left\langle-n_{j}\right\rangle}\right\rangle \equiv \tilde{a}_{j i l_{i}} .
$$

It means that the condition (37) holds for $l_{i}=1, \ldots, n_{i}, l_{j}=0, \ldots, n_{j}$.

Below we will show how to find the functions $\Psi_{i, n_{i}-l_{i}+1}(x)$ in (33). By construction, the scalar products (33) are elements of the unit matrix $I_{n}$, being a product of two $(n \times n)$-matrices: $M_{1} M_{2}=I_{n}$, where

$$
M_{1}=\left[\begin{array}{c}
\mathrm{d} X_{1, n_{1}} \\
\vdots \\
\mathrm{d} X_{1,1} \\
\vdots \\
\mathrm{d} X_{m, n_{m}} \\
\vdots \\
\mathrm{d} X_{m, 1}
\end{array}\right]
$$


and

$$
M_{2}=\left[\left(\frac{\partial}{\partial u_{1}}\right)^{\langle-1\rangle \pi} \cdots\left(\frac{\partial}{\partial u_{1}}\right)^{\left\langle-n_{1}\right\rangle \pi} \cdots\left(\frac{\partial}{\partial u_{m}}\right)^{\langle-1\rangle \pi} \cdots\left(\frac{\partial}{\partial u_{m}}\right)^{\left\langle-n_{m}\right\rangle \pi}\right]
$$

Since $M_{1}=M_{2}^{-1}$, we obtain the following rule to compute the new coordinates $X=\Psi(x)$ :

1. Compute $M_{2}^{-1}$, to get from its rows the differentials of the components of $\mathrm{d} X$.

2. Integrate them to get the coordinate transformation.

Example 10. Consider the non-reversible state equations

$$
x_{1}^{\langle 1\rangle}=x_{2} u_{1} u_{2}, \quad x_{2}^{\langle 1\rangle}=x_{1} / x_{5}, \quad x_{3}^{\langle 1\rangle}=x_{2}, \quad x_{4}^{\langle 1\rangle}=x_{5} u_{1}^{2} / x_{3}, \quad x_{5}^{\langle 1\rangle}=x_{2} u_{2} .
$$

Check first the linearizability conditions from Theorem 9. Observe that the choice $z_{1}=x_{1}, z_{2}=x_{4}$ results in the backward shift equations

$$
x_{1}^{\langle-1\rangle}=z_{1}^{\langle-1\rangle}, x_{2}^{\langle-1\rangle}=x_{3}, x_{3}^{\langle-1\rangle}=\frac{x_{1}^{2} z_{1}^{\langle-1\rangle}}{x_{2} x_{4} x_{5}^{2}}, x_{4}^{\langle-1\rangle}=z_{2}^{\langle-1\rangle}, x_{5}^{\langle-1\rangle}=\frac{z_{1}^{\langle-1\rangle}}{x_{2}}, u_{1}^{\langle-1\rangle}=\frac{x_{1}}{x_{5}}, u_{2}^{\langle-1\rangle}=\frac{x_{5}}{x_{3}} .
$$

Compute the differentials of the forward shifts of the state coordinates:

$$
\begin{aligned}
& \mathrm{d} x_{1}^{\langle 1\rangle}=u_{1} u_{2} \mathrm{~d} x_{2}+u_{2} x_{2} \mathrm{~d} u_{1}+u_{1} x_{2} \mathrm{~d} u_{2}, \quad \mathrm{~d} x_{2}^{\langle 1\rangle}=\frac{1}{x_{5}} \mathrm{~d} x_{1}-\frac{x_{1}}{x_{5}^{2}} \mathrm{~d} x_{5}, \quad \mathrm{~d} x_{3}^{\langle 1\rangle}=\mathrm{d} x_{2}, \\
& \mathrm{~d} x_{4}^{\langle 1\rangle}=-\frac{u_{1}^{2} x_{5}}{x_{3}^{2}} \mathrm{~d} x_{3}+\frac{u_{1}^{2}}{x_{3}} \mathrm{~d} x_{5}+\frac{2 u_{1} x_{5}}{x_{3}} \mathrm{~d} u_{1}, \quad \mathrm{~d} x_{5}^{\langle 1\rangle}=u_{2} \mathrm{~d} x_{2}+x_{2} \mathrm{~d} u_{2} .
\end{aligned}
$$

Next compute recursively, according to (7)-(9), the projections of the backward shifts of $\partial / \partial u_{i}, i=1,2$. For $i=1$ we get

$$
\begin{aligned}
\left(\frac{\partial}{\partial u_{1}}\right)^{\langle-1\rangle \pi} & =\sum_{i=1}^{n}\left\langle\mathrm{~d} x_{i}^{\langle 1\rangle}, \frac{\partial}{\partial u_{1}}\right\rangle^{\langle-1\rangle} \frac{\partial}{\partial x_{i}}=\left(u_{2} x_{2}\right)^{\langle-1\rangle} \frac{\partial}{\partial x_{1}}+\left(\frac{2 u_{1} x_{5}}{x_{3}}\right)^{\langle-1\rangle} \frac{\partial}{\partial x_{4}}=x_{5} \frac{\partial}{\partial x_{1}}+\frac{2 x_{4} x_{5}}{x_{1}} \frac{\partial}{\partial x_{4}} \\
\left(\frac{\partial}{\partial u_{1}}\right)^{\langle-2\rangle \pi} & =\sum_{i=1}^{n}\left\langle\mathrm{~d} x_{i}^{\langle 1\rangle},\left(\frac{\partial}{\partial u_{1}}\right)^{\langle-1\rangle}\right\rangle^{\langle-1\rangle} \frac{\partial}{\partial x_{i}}=\sum_{i=1}^{n}\left\langle\mathrm{~d} x_{i}^{\langle 1\rangle}, x_{5} \frac{\partial}{\partial x_{1}}\right\rangle^{\langle-1\rangle} \frac{\partial}{\partial x_{i}} \frac{\partial}{\partial x_{2}} \\
\left(\frac{\partial}{\partial u_{1}}\right)^{\langle-3\rangle \pi} & =\sum_{i=1}^{n}\left\langle\mathrm{~d} x_{i}^{\langle 1\rangle},\left(\frac{\partial}{\partial u_{1}}\right)^{\langle-2\rangle}\right\rangle^{\langle-1\rangle} \frac{\partial}{\partial x_{i}}=\sum_{i=1}^{n}\left\langle\mathrm{~d} x_{i}^{\langle 1\rangle}, \frac{\partial}{\partial x_{2}}\right\rangle^{\langle-1\rangle} \frac{\partial}{\partial x_{i}} \\
& =\left(u_{1} u_{2}\right)^{\langle-1\rangle} \frac{\partial}{\partial x_{1}}+\frac{\partial}{\partial x_{3}}+u_{2}^{\langle-1\rangle} \frac{\partial}{\partial x_{5}}=\frac{x_{1}}{x_{3}} \frac{\partial}{\partial x_{1}}+\frac{\partial}{\partial x_{3}}+\frac{x_{5}}{x_{3}} \frac{\partial}{\partial x_{5}} .
\end{aligned}
$$

Analogously, we find

$$
\left(\frac{\partial}{\partial u_{2}}\right)^{\langle-1\rangle \pi}=\frac{x_{1} x_{3}}{x_{5}} \frac{\partial}{\partial x_{1}}+x_{3} \frac{\partial}{\partial x_{5}},\left(\frac{\partial}{\partial u_{2}}\right)^{\langle-2\rangle \pi}=\frac{x_{1}^{2}}{x_{5}^{2}} \frac{\partial}{\partial x_{4}},\left(\frac{\partial}{\partial u_{2}}\right)^{\langle-3\rangle \pi}=0 .
$$

Observe, that the non-zero vector fields $\left(\partial / \partial u_{i}\right)^{\langle-l\rangle}, i=1,2, l=1,2,3$, define the basis vector fields of $\operatorname{span}_{\mathscr{K}}\{\partial / \partial x\}$ and their coefficients depend only on the variable $x$. Then, according to Theorem 9 , the state 
equations (40) are state linearizable. To find the state transformation, construct first

$$
\begin{aligned}
M_{2}= & {\left[\left(\frac{\partial}{\partial u_{1}}\right)^{\langle-1\rangle \pi}\left(\frac{\partial}{\partial u_{2}}\right)^{\langle-1\rangle \pi}\left(\frac{\partial}{\partial u_{1}}\right)^{\langle-2\rangle \pi}\left(\frac{\partial}{\partial u_{2}}\right)^{\langle-2\rangle \pi}\left(\frac{\partial}{\partial u_{1}}\right)^{\langle-3\rangle \pi}\right] } \\
= & \left(\begin{array}{ccccc}
x_{5} & x_{1} x_{3} / x_{5} & 0 & 0 & x_{1} / x_{3} \\
0 & 0 & 1 & 0 & 0 \\
0 & 0 & 0 & 0 & 1 \\
2 x_{4} x_{5} / x_{1} & 0 & 0 & x_{1}^{2} / x_{5}^{2} & 0 \\
0 & x_{3} & 0 & 0 & x_{5} / x_{3}
\end{array}\right)
\end{aligned}
$$

Compute next $M_{1}=M_{2}^{-1}$ :

$$
M_{1}=\left[\begin{array}{l}
\mathrm{d} X_{1,3} \\
\mathrm{~d} X_{2,2} \\
\mathrm{~d} X_{1,2} \\
\mathrm{~d} X_{2,1} \\
\mathrm{~d} X_{1,1}
\end{array}\right]=\left(\begin{array}{ccccc}
\frac{1}{x_{5}} & 0 & 0 & 0 & -\frac{x_{1}}{x_{5}^{2}} \\
0 & 0 & -\frac{x_{5}}{x_{3}^{2}} & 0 & \frac{1}{x_{3}} \\
0 & 1 & 0 & 0 & 0 \\
-\frac{2 x_{4} x_{5}^{2}}{x_{1}^{3}} & 0 & 0 & \frac{x_{5}^{2}}{x_{1}^{2}} & \frac{2 x_{4} x_{5}}{x_{1}^{2}} \\
0 & 0 & 1 & 0 & 0
\end{array}\right) .
$$

In order to define the new coordinates (33), integrate the 1-forms $\mathrm{d} X_{i, l}$, given in the form of the row vectors as the rows of matrix $M_{1}$. This leads to the state transformation $X_{1,1}=x_{3}, X_{1,2}=x_{2}, X_{1,3}=x_{1} / x_{5}, X_{2,1}=$ $\left(x_{4} x_{5}^{2}\right) / x_{1}^{2}, X_{2,2}=x_{5} / x_{3}$. The state equations read in the new coordinates $X_{1,1}^{\langle 1\rangle}=X_{1,2}, X_{1,2}^{\langle 1\rangle}=X_{1,3}, X_{1,3}^{\langle 1\rangle}=u_{1}$, $X_{2,1}^{\langle 1\rangle}=X_{2,2}, X_{2,2}^{\langle 1\rangle}=u_{2}$.

\section{COMPARISON WITH THE FEEDBACK LINEARIZABILITY CONDITIONS}

We compare the conditions of Theorem 9 with those for static state feedback linearizability from [1], recalled in Proposition 11 below. Note that the paper [1] addresses the problem, using the same tools that are applied in this paper. The goal of the comparison is to show how exactly feedback allows to relax the state linearizability conditions.

A regular static state feedback is an analytic mapping $u=\alpha(x, v), v \in \mathbb{R}^{m}$, such that $\operatorname{rank}_{\mathscr{K}}(\partial \alpha / \partial v)=m$. System (1) is said to be (generically) linearizable by a regular static state feedback if there exists a state transformation $X=\Psi(x)$ and a regular static state feedback such that the system in the new coordinates takes the Brunovsky form

$$
X_{i, 1}^{\langle 1\rangle}=X_{i, 2}, \ldots, \quad X_{i, n_{i}-1}^{\langle 1\rangle}=X_{i, n_{i}}, \quad X_{i, n_{i}}^{\langle 1\rangle}=v_{i}, \quad i=1, \ldots, m .
$$

Note that using the Brunovsky form (41) does not lead to the loss of generality compared with the linear accessible system (18) in Theorem 9 because the equations (18) are always transformable into the form (41) by state transformation and regular static state feedback, see e.g. [9]. 
Recall that system (1) is (generically) static state feedback linearizable if and only if (i) $\mathscr{D}_{k}, k=1, \ldots, k^{\star}$, are involutive and (ii) $\operatorname{dim}_{\mathscr{K}} \mathscr{D}_{k^{\star}}^{\pi}=n$ [1]. For easier comparison, rewrite this result as in the proposition below.

Proposition 11. System (1) is (generically) static state feedback linearizable if and only if $\left(a^{\prime}\right) \mathscr{D}_{k}^{\pi}, k=1, \ldots, k^{\star}$, are involutive,

$\left(b^{\prime}\right) \mathscr{D}_{k}^{\pi}, k=1, \ldots, k^{\star}$, are invariant under taking the Lie brackets with $\partial / \partial z_{i}^{\langle-1\rangle}$, i.e. for $i, j=1, \ldots, m$ and $l_{j}=1, \ldots, k$,

$$
\left[\frac{\partial}{\partial z_{i}^{\langle-1\rangle}},\left(\frac{\partial}{\partial u_{j}}\right)^{\left\langle-l_{j}\right\rangle \pi}\right] \in \mathscr{D}_{k}^{\pi}
$$

and $\left(c^{\prime}\right) \operatorname{dim}_{\mathscr{K}} \mathscr{D}_{k^{\star}}^{\pi}=n$.

Proof. One has to show that involutivity of $\mathscr{D}_{k}, k=1, \ldots, k^{\star}$, is equivalent to $\left(\mathrm{a}^{\prime}\right)$ and $\left(\mathrm{b}^{\prime}\right)$. According to (10), the involutivity conditions of $\mathscr{D}_{k}$ can be rewritten for $i, j=1, \ldots, m$ and $l_{i}, l_{j}=1, \ldots, k$ as:

$$
\begin{aligned}
& {\left[\left(\frac{\partial}{\partial u_{i}}\right)^{\left\langle-l_{i}\right\rangle \pi},\left(\frac{\partial}{\partial u_{j}}\right)^{\left\langle-l_{j}\right\rangle \pi}\right] \in \mathscr{D}_{k},} \\
& {\left[\frac{\partial}{\partial z_{i}^{\langle-1\rangle}},\left(\frac{\partial}{\partial u_{j}}\right)^{\left\langle-l_{j}\right\rangle \pi}\right] \in \mathscr{D}_{k} .}
\end{aligned}
$$

We will show first that $\mathscr{D}_{k}^{\pi}$ is involutive if and only if (43) holds. Due to (11), $\mathscr{D}_{k}^{\pi}$ is involutive if and only if

$$
\left[\left(\frac{\partial}{\partial u_{i}}\right)^{\left\langle-l_{i}\right\rangle \pi},\left(\frac{\partial}{\partial u_{j}}\right)^{\left\langle-l_{j}\right\rangle \pi}\right] \in \mathscr{D}_{k}^{\pi}
$$

and therefore, we need to prove that (43) is equivalent to (45). Observe that the projections $\left(\partial / \partial u_{i}\right)^{\left\langle-l_{i}\right\rangle \pi} \in$ $\operatorname{span}_{\mathscr{K}}\{\partial / \partial x\}$ and thus also their Lie brackets belong to this vector space. Consequently, from (43) follows (45). The inverse is obvious because $\mathscr{D}_{k}^{\pi} \subset \mathscr{D}_{k}$.

Next we will show that (42) is equivalent to (44). Since $\left(\partial / \partial u_{j}\right)^{\left\langle-l_{j}\right\rangle \pi} \in \operatorname{span}_{\mathscr{K}}\{\partial / \partial x\}$, it can be written as $\sum_{q=1}^{n} \alpha_{q j l_{j}} \partial / \partial x_{l}$. Then

$$
\left[\frac{\partial}{\partial z_{i}^{\langle-1\rangle}},\left(\frac{\partial}{\partial u_{j}}\right)^{\left\langle-l_{j}\right\rangle \pi}\right]=\sum_{q=1}^{n} \frac{\partial \alpha_{q j l_{j}}}{\partial z_{i}^{\langle-1\rangle}} \frac{\partial}{\partial x_{q}} \in \operatorname{span}_{\mathscr{K}}\left\{\frac{\partial}{\partial x}\right\} .
$$

Therefore, if (44) is satisfied, (42) also holds. The inverse is obvious.

Compare now the conditions of state linearizability given in Theorem 9 and those from Proposition 11. We suppose that the static state feedback linearizability conditions from Proposition 11 hold and examine which additional restrictions are necessary for system (1) to be state linearizable. First, due to Remark 2 , the condition (a) of Theorem 9 is equivalent to the condition $\left(\mathrm{c}^{\prime}\right)$ of Proposition 11.

Though the conditions of Theorem 9 are easy to check (no need to compute the Lie brackets of vector fields), for comparison with the static state feedback linearizability conditions it is important to recall from the proof of the theorem that the condition (b) is equivalent to the condition (28), and the latter implies, by Lemma 6, the condition (29).

Compare now the condition $\left(b^{\prime}\right)$ of Proposition 11 with (28). Suppose that $\left(b^{\prime}\right)$, given by (42), holds and show which additional restrictions are required when the feedback is not allowed. From $\left(b^{\prime}\right)$ follows 
that the distributions $\mathscr{D}_{k}^{\pi}, k=1, \ldots, k^{\star}$, are invariant under taking the Lie brackets with respect to $\partial / \partial z^{\langle-1\rangle}$ (recall that $\left.k^{\star}=\max \left\{n_{i}\right\}\right)$. Since by (9) the vector fields $\left(\partial / \partial u_{i}\right)^{\left\langle-l_{i}\right\rangle}, i=1, \ldots, m, l_{i}=1, \ldots, n_{i}+1$, are the basis vector fields of $\mathscr{D}_{k}^{\pi}, k=1, \ldots, k^{\star}+1$, the corresponding state linearizability condition (28) has two supplementary restrictions. First, it requires that the above condition holds also for $k=k^{\star}+1$. Second, (28) requires that the Lie brackets of the vector fields $\left(\partial / \partial u_{i}\right)^{\left\langle-l_{i}\right\rangle}$ with $\partial / \partial z^{\langle-1\rangle}$ must be identically equal to zero, i.e. the coefficients of $\left(\partial / \partial u_{i}\right)^{\left\langle-l_{i}\right\rangle}$ depend only on $x$, which is not the case for $\left(\mathrm{b}^{\prime}\right)$.

Next consider the condition $\left(\mathrm{a}^{\prime}\right)$ from Proposition 11, given by (45) and requiring that the distributions $\mathscr{D}_{k}^{\pi}, k=1, \ldots, k^{\star}$, must be involutive. The corresponding condition in the case of a state linearizable system is (29), which is also more restrictive than (45), having two additional requirements. First, it also holds again for $k=k^{\star}+1$ and second, it requires not only the involutivity of the distributions $\mathscr{D}_{k}^{\pi}, k=1, \ldots, k^{\star}+1$, but also the commutativity of all the basis vector fields $\left(\partial / \partial u_{i}\right)^{\left\langle-l_{i}\right\rangle \pi}, i=1, \ldots, m, l_{i}=1, \ldots, n_{i}+1$, of these distributions. However, recall that, due to Lemma 6, if (28) holds, then (29) is automatically satisfied. For a static state feedback linearizable system the analogous condition (45) does not follow from (42) and must be checked separately. Nevertheless, if (28) holds, (45) is also satisfied.

To resume, if we do not allow the feedback, then two additional restrictions are required: for $i, j=$ $1, \ldots, m, l_{i}=1, \ldots, n_{i}+1, l_{j}=1, \ldots, n_{j}+1$,

$$
\text { (1) }\left[\left(\frac{\partial}{\partial u_{i}}\right)^{\left\langle-l_{i}\right\rangle \pi},\left(\frac{\partial}{\partial u_{j}}\right)^{\left\langle-l_{j}\right\rangle \pi}\right] \equiv 0 \text {, }
$$

and

(2) $\left[\frac{\partial}{\partial z_{i}^{\langle-1\rangle}},\left(\frac{\partial}{\partial u_{j}}\right)^{\left\langle-l_{j}\right\rangle \pi}\right] \equiv 0$.

Example 12. Consider the dynamical system

$$
x_{1}^{\langle 1\rangle}=\frac{x_{2}}{x_{1}}, \quad x_{2}^{\langle 1\rangle}=\frac{u_{1} x_{2}}{x_{1}}, \quad x_{3}^{\langle 1\rangle}=\frac{u_{2} x_{2} x_{3}}{x_{1}^{2}} .
$$

Defining $z_{1}=x_{1}, z_{2}=u_{2}$, we get the extended state equations, resulting in the backward shift equations

$$
x_{1}^{\langle-1\rangle}=z_{1}^{\langle-1\rangle}, \quad x_{2}^{\langle-1\rangle}=z_{1}^{\langle-1\rangle} x_{1}, \quad x_{3}^{\langle-1\rangle}=\frac{z_{1}^{\langle-1\rangle} x_{3}}{x_{1} z_{2}^{\langle-1\rangle}} .
$$

Compute the differentials of the forward shifts of the state coordinates

$$
\begin{aligned}
\mathrm{d} x_{1}^{\langle 1\rangle} & =-\frac{x_{2}}{x_{1}^{2}} \mathrm{~d} x_{1}+\frac{1}{x_{1}} \mathrm{~d} x_{2}, \\
\mathrm{~d} x_{2}^{\langle 1\rangle} & =-\frac{u_{1} x_{2}}{x_{1}^{2}} \mathrm{~d} x_{1}+\frac{u_{1}}{x_{1}} \mathrm{~d} x_{2}+\frac{x_{2}}{x_{1}} \mathrm{~d} u_{1}, \\
\mathrm{~d} x_{3}^{\langle 1\rangle} & =-\frac{2 u_{2} x_{2} x_{3}}{x_{1}^{3}} \mathrm{~d} x_{1}+\frac{u_{2} x_{3}}{x_{1}^{2}} \mathrm{~d} x_{2}+\frac{u_{2} x_{2}}{x_{1}^{2}} \mathrm{~d} x_{3}+\frac{x_{2} x_{3}}{x_{1}^{2}} \mathrm{~d} u_{2},
\end{aligned}
$$

and the projections of the backward shifts of the vector fields $\partial / \partial u_{i}$ according to (7), (8) and (9), taking into account also (48): 


$$
\begin{aligned}
\left(\frac{\partial}{\partial u_{1}}\right)^{\langle-1\rangle \pi} & =\sum_{i=1}^{3}\left\langle\mathrm{~d} x_{i}^{\langle 1\rangle}, \frac{\partial}{\partial u_{1}}\right\rangle^{\langle-1\rangle} \frac{\partial}{\partial x_{i}}=\left(\frac{x_{2}}{x_{1}}\right)^{\langle-1\rangle} \frac{\partial}{\partial x_{2}}=x_{1} \frac{\partial}{\partial x_{2}} \\
\left(\frac{\partial}{\partial u_{2}}\right)^{\langle-1\rangle \pi} & =\sum_{i=1}^{3}\left\langle\mathrm{~d} x_{i}^{\langle 1\rangle}, \frac{\partial}{\partial u_{2}}\right\rangle^{\langle-1\rangle} \frac{\partial}{\partial x_{i}}=\left(\frac{x_{2} x_{3}}{x_{1}^{2}}\right)^{\langle-1\rangle} \frac{\partial}{\partial x_{3}}=\frac{x_{3}}{z_{2}^{\langle-1\rangle} \frac{\partial}{\partial x_{3}}} \\
\left(\frac{\partial}{\partial u_{1}}\right)^{\langle-2\rangle \pi} & =\sum_{i=1}^{3}\left\langle\mathrm{~d} x_{i}^{\langle 1\rangle},\left(\frac{\partial}{\partial u_{1}}\right)^{\langle-1\rangle}\right\rangle^{\langle-1\rangle} \frac{\partial}{\partial x_{i}} \\
& =\frac{\partial}{\partial x_{1}}+u_{1}\langle-1\rangle \frac{\partial}{\partial x_{2}}+\left(\frac{u_{2} x_{3}}{x_{1}^{2}}\right)^{\langle-1\rangle} \frac{\partial}{\partial x_{3}}=\frac{\partial}{\partial x_{1}}+\frac{x_{2}}{x_{1}} \frac{\partial}{\partial x_{2}}+\frac{x_{3}}{x_{1}} \frac{\partial}{\partial x_{3}}
\end{aligned}
$$

Since the vector fields (49) already span the entire space $\operatorname{span}_{\mathscr{K}}\{\partial / \partial x\}$, further computations do not yield additional linearly indepent vector fields. According to (11) we get the distributions

$$
\begin{aligned}
\mathscr{D}_{1}^{\pi} & =\operatorname{span}_{\mathscr{K}}\left\{x_{1} \frac{\partial}{\partial x_{2}}, \frac{x_{3}}{z_{2}^{\langle-1\rangle}} \frac{\partial}{\partial x_{3}}\right\}, \\
\mathscr{D}_{2}^{\pi} & =\operatorname{span}_{\mathscr{K}}\left\{x_{1} \frac{\partial}{\partial x_{2}}, \frac{x_{3}}{z_{2}^{\langle-1\rangle}} \frac{\partial}{\partial x_{3}}, \frac{\partial}{\partial x_{1}}+\frac{x_{2}}{x_{1}} \frac{\partial}{\partial x_{2}}+\frac{x_{3}}{x_{1}} \frac{\partial}{\partial x_{3}}\right\} .
\end{aligned}
$$

Check first the static state feedback linearizability conditions from Proposition 11. One can easily see that the Lie brackets of the basis vector fields of $\mathscr{D}_{1}^{\pi}\left(\mathscr{D}_{2}^{\pi}\right)$ are either zero or belong to $\mathscr{D}_{1}^{\pi}\left(\mathscr{D}_{2}^{\pi}\right)$. Consequently, the condition ( $\left.\mathrm{a}^{\prime}\right)$ holds for $\mathscr{D}_{1}^{\pi}$ and $\mathscr{D}_{2}^{\pi}$. Computing the Lie brackets of the basis vector fields of $\mathscr{D}_{1}^{\pi}$ and $\mathscr{D}_{2}^{\pi}$ with respect to $\partial / \partial z_{1}^{\langle-1\rangle}$ shows that $\left(\mathrm{b}^{\prime}\right)$ is satisfied. Next, $\operatorname{dim}_{\mathscr{K}} \mathscr{D}_{2}^{\pi}=3$, and therefore, $\left(\mathrm{c}^{\prime}\right)$ is also true. The system is static state feedback linearizable. Note that the application of the state transformation $X_{1,1}=x_{1}$, $X_{1,2}=x_{2} / x_{1}, X_{2,1}=x_{3}$, and the state feedback $u_{1}=v_{1}, u_{2}=v_{2} x_{1}^{2} /\left(x_{2} x_{3}\right)$ results in the linear equations

$$
X_{1,1}^{\langle 1\rangle}=X_{1,2}, \quad X_{1,2}^{\langle 1\rangle}=v_{1}, \quad X_{2,1}^{\langle 1\rangle}=v_{2} .
$$

Next check state linearizability. The condition (a) of Theorem 9 is satisfied with $n_{1}=2, n_{2}=1$. Although the distributions $\mathscr{D}_{1}^{\pi}$ and $\mathscr{D}_{2}^{\pi}$ are invariant under taking the Lie derivatives with respect to $\partial / \partial z^{\langle-1\rangle}$, for state linearizability the Lie brackets of the vector fields (49) with $\partial / \partial z^{\langle-1\rangle}$ must be identically equal to zero, see (47). However, for the vector field $\left(\partial / \partial u_{2}\right)^{\langle-1\rangle}$, given by (49), this is obviously not the case. Therefore, the system is not state linearizable.

Finally, note that static state feedback linearization as an iconic research problem has been studied, using different approaches for different discrete-time nonlinear system classes. In [10] and [11] the problem is addressed by applying another algebraic approach, the so-called functions' algebra (FA), by working directly with functions, defined by system equations and not with the vector spaces of the 1 -forms or vector fields like in [2] and [1], respectively. The solvability conditions based on FA were compared with those in [2] for analytic systems in [10]. Moreover, the conditions of [2] were compared with those recalled in this paper in [1]. Thus, by combining these two comparisons, one may conclude that at least for analytic systems addressed in this paper, the FA approach provides no advantages. For this reason it is probably not important to find the analogues of the additional conditions (46) and (47) in this paper by means of the FA approach, though, in principle, it is possible. In [12] it is shown how to use the vector spaces of differential 1 -forms to formalize and simplify the computations in the smooth case. Of course, the FA approach allows to address also non-smooth systems, though the computations in the non-smooth case are difficult, see [12]. In a similar manner (but for continuous-time case) the distributions of vector fields have been used to assist the computations, see for instance [13]. It would be more interesting to compare the solvability conditions from [10] with the conditions when state feedback is not allowed within the FA formalism to address the non-smooth case. However, as shown in [12], computations in the non-smooth case are difficult to handle. 


\section{CONCLUSION}

The present paper is devoted to the linearizability problem of discrete-time nonlinear control systems via state transformation. The necessary and sufficient conditions for the existence of such state transformation are formulated in terms of the backward shifts of the vector fields, defined by system dynamics, and the rule for finding the corresponding state transformation is given. Next, the relationship of these conditions with static state feedback linearizability conditions, which are also given in terms of backward shifts of vector fields, is examined.

Since our linearizability conditions are formulated in a form that allows, by direct inspection, to see how the conditions will be relaxed if also state feedback is allowed, we believe that the conditions will be helpful in finding the conditions under which one can transform the state equations directly into the classical or extended observer forms without finding the input-output equations, as carried out, for example, in [14]. It should be noted that such conditions are still missing in case the state equations are not state reversible. It is important to recall that such observer forms consist of linear equations up to nonlinear input/output injection terms, which depend only on inputs and outputs in the case of the classical observer form, but also on their backward shifts in the case of the extended observer form.

\section{ACKNOWLEDGEMENTS}

The work of Ü. Kotta was partially supported by the Estonian Centre of Excellence in IT, funded by the European Regional Development Fund. The publication costs of this article were covered by the Estonian Academy of Sciences.

\section{REFERENCES}

1. Mullari, T., Kotta, Ü., Bartosiewicz, Z., Pawluszewicz, E., and Moog, C. H. Forward and backward shifts of vector fields: towards the dual algebraic framework. IEEE Trans. Automat. Contr., 2017, 62(6), 3029-3033.

2. Aranda-Bricaire, E., Kotta, Ü., and Moog, C. H. Linearization of discrete-time systems. SIAM J. Control Optim., 1996, 34(6), 1999-2023.

3. Belikov, J., Kaldmäe, A., and Kotta, Ü. Global linearization approach to nonlinear control systems: a brief tutorial overview. Proc. Estonian Acad. Sci., 2017, 66(3), 243-263.

4. Lee, A. G., Arapostathis, A., and Marcus, S. I. Linearization of discrete-time systems. Int. J. Control, 1987, 45(5), $1803-1822$.

5. Grizzle, J. V. Feedback linearization of discrete-time systems. Lect. Notes Control Inf. Sci., 1986, 83, 273-281.

6. Nam, K. Linearization of discrete-time systems and a canonical structure. IEEE Trans. Automat. Contr., 1989, 34(1), 119-122.

7. Conte, G., Moog, C. H., and Perdon, A. M. Nonlinear Control Systems: an Algebraic Setting. Springer, London, 1999.

8. Nijmeijer, H. and van der Schaft, A. J. Nonlinear Dynamical Control Systems, Springer, New York, 1990.

9. Kailath, T. Linear Systems, Prentice-Hall, Inc., Upper Saddle River, 1980.

10. Kotta, Ü., Tõnso, M., Shumsky, A., and Zhirabok, A. Feedback linearization and lattice theory. Syst. Control Lett., 2013, 62(3), $248-255$.

11. Kaldmäe, A., Kotta, Ü., Shumsky, A., and Zhirabok, A. Feedback linearization of possibly non-smooth systems. Proc. Estonian Acad. Sci., 2017, 66(2), 109-123.

12. Belikov, J., Kaldmäe, A., Kaparin, V., Shumsky, A., Kotta, Ü., Tõnso, M., and Zhirabok, A. Functions' algebra in nonlinear control: computational aspects and software. Proc. Estonian Acad. Sci., 2017, 66(1), 89-107.

13. Kotta, Ü. and Mullari, T. Discussion on: "Unified approach to the problem of full decoupling via output feedback". Eur. J. Control, 2010, 16(4), 326-328.

14. Kaparin, V. and Kotta, Ü. Transformation of nonlinear MIMO discrete-time systems into the extended observer form. Asian J. Control, 2019, 21(5), 2202-2217. 
Define $n$ linearly independent 1 -forms $\omega_{i}=\sum_{l=1}^{n} \alpha_{i l}(x) \mathrm{d} x_{l}, i=1, \ldots, n$, such that

$$
\left\langle\omega_{i}, \Xi_{j}\right\rangle \equiv \delta_{i j}
$$

This is possible since the vector fields $\Xi_{j}, j=1, \ldots, n$, being linearly independent, can be understood as column vectors of a non-singular matrix. The rows of its inverse matrix define the 1-forms in (51). We will prove that under the commutativity assumption of $\Xi_{j}$, all $\omega_{i}$ are exact, i.e. there exist $n$ independent functions $\Psi_{i}(x)$ such that $\omega_{i}=\mathrm{d} \Psi_{i}$, or equivalently, the exterior differentials $\mathrm{d} \omega_{i} \equiv 0$. Recall that $\mathrm{d} \omega_{i}$ is the 2-form

$$
\mathrm{d} \omega_{i}=\sum_{j=1}^{l} \sum_{l=2}^{n}\left(\frac{\partial \alpha_{i l}}{\partial x_{j}}-\frac{\partial \alpha_{i j}}{\partial x_{l}}\right) \mathrm{d} x_{j} \wedge \mathrm{d} x_{l}
$$

and so from $\mathrm{d} \omega_{i} \equiv 0$ we get

$$
\frac{\partial \alpha_{i l}}{\partial x_{j}}-\frac{\partial \alpha_{i j}}{\partial x_{l}} \equiv 0, \quad i, j, l=1, \ldots, n
$$

The scalar product (51) can be rewritten as

$$
\sum_{l=1}^{n} \alpha_{i l}(x) \xi_{l j}(x) \equiv \delta_{i j}
$$

Taking the partial derivative of this equality with respect to $x_{k}$, we obtain

$$
\sum_{l=1}^{n} \frac{\partial \alpha_{i l}}{\partial x_{k}} \xi_{l j}=-\sum_{l=1}^{n} \alpha_{i l} \frac{\partial \xi_{l j}}{\partial x_{k}}
$$

Recall next that the Lie derivative of a scalar function $\phi(x)$ with respect to the vector field $\Xi=\sum_{i=1}^{n} \xi_{i}(x)$ $\left(\partial / \partial x_{i}\right)$ is defined as

$$
L_{\Xi} \phi=\sum_{i=1}^{n} \frac{\partial \phi(x)}{\partial x_{i}} \xi_{i}(x)
$$

Compute, according to (56), the Lie derivative of the scalar product (54) with respect to the vector field $\Xi_{k}$. This gives the first and the second terms in (57) below. Next we add and subtract the third and the fourth term which are equal, but with opposite signs, which allows to rewrite later the Lie derivative in a suitable form

$$
L_{\Xi_{k}}\left\langle\omega_{i}, \Xi_{j}\right\rangle=\sum_{l, q=1}^{n}\left(\frac{\partial \alpha_{i l}}{\partial x_{q}} \xi_{l j} \xi_{q k}+\alpha_{i l} \frac{\partial \xi_{l j}}{\partial x_{q}} \xi_{q k}+\alpha_{i l} \frac{\partial \xi_{l k}}{\partial x_{q}} \xi_{q j}-\alpha_{i l} \frac{\partial \xi_{l k}}{\partial x_{q}} \xi_{q j}\right) .
$$

Due to the definition of the Lie brackets

$$
\left[\Xi_{k}, \Xi_{j}\right]=\sum_{l, q=1}^{n}\left(\frac{\partial \xi_{l j}}{\partial x_{q}} \xi_{q k}-\frac{\partial \xi_{l k}}{\partial x_{q}} \xi_{q j}\right) \frac{\partial}{\partial x_{q}}
$$

and from the definition of the scalar product we obtain

$$
\left\langle\omega_{i},\left[\Xi_{k}, \Xi_{j}\right]\right\rangle=\sum_{l, q=1}^{n} \alpha_{i l}\left(\frac{\partial \xi_{l j}}{\partial x_{q}} \xi_{q k}-\frac{\partial \xi_{l k}}{\partial x_{q}} \xi_{q j}\right)
$$


The left hand side of (59) is identically equal to zero according to the commutativity of $\Xi_{i}$ and $\Xi_{j}$. Observe that the right hand side of (59) is exactly the sum of the second and the fourth terms in (57). Therefore, (57) takes the simpler form

$$
\sum_{l, q=1}^{n}\left(\frac{\partial \alpha_{i l}}{\partial x_{q}} \xi_{l j} \xi_{q k}+\alpha_{i l} \frac{\partial \xi_{l k}}{\partial x_{q}} \xi_{q j}\right) \equiv 0
$$

The second term on the left hand side of (60) can be rewritten, according to (55), as

$$
\sum_{l, q=1}^{n} \alpha_{i l} \frac{\partial \xi_{l k}}{\partial x_{q}} \xi_{q j}=-\sum_{l, q=1}^{n} \frac{\partial \alpha_{i l}}{\partial x_{q}} \xi_{l k} \xi_{q j}
$$

Substituting (61) into (60) and exchanging the summation indices $l$ and $q$ in the second term, we obtain

$$
\sum_{l, q=1}^{n}\left(\frac{\partial \alpha_{i l}}{\partial x_{q}}-\frac{\partial \alpha_{i q}}{\partial x_{l}}\right) \xi_{l j} \xi_{q k} \equiv 0, \quad i, j, k=1, \ldots n .
$$

The above equality can be interpreted as the product of three $(n \times n)$-dimensional matrices

$$
P^{T} Q_{i} P \equiv 0_{n, n},
$$

where $0_{n, n}$ is the $(n \times n)$-dimensional zero matrix and the matrices $P$ and $Q_{i}$ are defined as

$$
P_{l j}=\xi_{l j}, \quad Q_{i, l q}=\frac{\partial \alpha_{i l}}{\partial x_{q}}-\frac{\partial \alpha_{i q}}{\partial x_{l}}
$$

We show next that all elements of $Q_{i}$ are identically zero. The matrix $P^{T} Q_{i} P$ in (62) is a zero matrix and therefore $\operatorname{rank}_{\mathscr{K}}\left(P^{T} Q_{i} P\right) \equiv 0$. Due to the Sylvester formula, $\operatorname{rank}_{\mathscr{K}}\left(P^{T} Q_{i} P\right)=\min \left(\operatorname{rank}_{\mathscr{K}} Q_{i}, \operatorname{rank}_{\mathscr{K}} P\right)$. As $\operatorname{rank}_{\mathscr{K}} P=n$ due to linear independence of $\Xi_{j}$, we get $\operatorname{rank}_{\mathscr{K}} Q_{i}=0$. Since only the zero matrix has the rank zero, (53) holds for all $i, j, l$, and so $\omega_{i}, i=1, \ldots, n$, are exact. By $(16) \Psi_{i}(x), i=1, \ldots, n$, are independent functions and therefore they define the coordinate transformation $X_{i}=\Psi_{i}(x)$.

Next, let us express the scalar product (16) in terms of the new coordinates $X$, taking into account that 1) due to (14) $\left.\mathrm{d} \Psi_{i}=\mathrm{d} X_{i}, 2\right)$ the vector field $\Xi_{i}$ transforms into the vector field $\Psi_{*} \Xi_{i}$ according to (15), and 3 ) the coordinate transformation does not change the value of a constant. We get, in the new coordinates,

$$
\left\langle\mathrm{d} X_{i}, \Psi_{*} \Xi_{j}\right\rangle \equiv \delta_{i j}, \quad i, j=1, \ldots, n .
$$

Comparing (65) with the obvious equality $\left\langle\mathrm{d} X_{i}, \partial / \partial X_{j}\right\rangle \equiv \delta_{i j}$, we obtain (17).

\section{THE PROOF OF LEMMA 4}

APPENDIX B

In principle, the proof of this lemma can be performed by induction. Since the $k$-th step is technically extremely complicated to follow, we prove the first and the second steps instead. The continuation is obvious.

We first examine the case $l=1$. Observe that by (7) and (8) the vector field $\left(\partial / \partial u_{i}\right)^{\langle-1\rangle}$ has no components in the directions of $\partial / \partial u^{\langle k\rangle}, k \geq 0$, and $\partial / \partial z^{\langle-l\rangle}, l>1$. Really, all the coefficients $b_{j k}$ in (8) are zero because $\left\langle\mathrm{d} u_{j}^{\langle k+1\rangle}, \partial / \partial u_{i}\right\rangle \equiv 0$ for all $j=1, \ldots, m$ and $k \geq 0$. Moreover, also the coefficients $c_{q l} \equiv 0$ for $l \geq 2$, because $\left\langle\mathrm{d} z_{q}^{\langle-l+1\rangle}, \partial / \partial u_{i}\right\rangle \equiv 0$ for $l \geq 2$. Therefore, according to (7), (8), (1) and (2)

$$
\left(\frac{\partial}{\partial u_{i}}\right)^{\langle-1\rangle}=\sum_{j_{1}=1}^{n}\left(\frac{\partial \bar{\Phi}_{j_{1}}}{\partial u_{i}}\right)^{\langle-1\rangle} \frac{\partial}{\partial x_{j_{1}}}+\sum_{p_{1}=1}^{m}\left(\frac{\partial \chi_{p_{1}}}{\partial u_{i}}\right)^{\langle-1\rangle} \frac{\partial}{\partial z_{p_{1}}^{\langle-1\rangle}} .
$$


From (66) follows that for $l=1$ the property (i) is satisfied. Next, since the functions $\bar{\Phi}$ and $\chi$ in (1) and (2) depend only on the variables $x$ and $u$, their partial derivatives are also the functions of $x$ and $u$ only. By (3), the backward shifts of partial derivatives depend only on $x$ and $z^{\langle-1\rangle}$. Hence, the coefficients of the vector field (66) are the functions of $x$ and $z^{\langle-1\rangle}$ only and (ii) holds.

We show next that the lemma is valid for $l=2$. Again, one can easily show that $\left(\partial / \partial u_{i}\right)^{\langle-2\rangle}$ has no components in the directions of the vector fields $\partial / \partial u_{j}^{\langle k\rangle}, k \geq 0$, and $\partial / \partial z_{q}^{\langle-l\rangle}, l>2$. Namely, taking $\Xi=\left(\partial / \partial u_{i}\right)^{\langle-1\rangle}$ from (66) and substituting it into (8), all the coefficients $b_{i k}$ and $c_{q l}, l>2$, are identically equal to zero. Shift the vector field $\left(\partial / \partial u_{i}\right)^{\langle-1\rangle}$ backward, using (7), (8) and (66) to get

$$
\begin{aligned}
\left(\frac{\partial}{\partial u_{i}}\right)^{\langle-2\rangle}= & \sum_{j_{2}=1}^{n}\left\langle\mathrm{~d} x_{j_{2}}^{\langle 1\rangle},\left(\frac{\partial}{\partial u_{i}}\right)^{\langle-1\rangle}\right\rangle^{\langle-1\rangle} \frac{\partial}{\partial x_{j_{2}}}+\sum_{p_{2}=1}^{m}\left\langle\mathrm{~d} z_{p_{1}},\left(\frac{\partial}{\partial u_{i}}\right)^{\langle-1\rangle}\right\rangle^{\langle-1\rangle} \frac{\partial}{\partial z_{p_{2}}^{\langle-1\rangle}} \\
& +\sum_{p_{2}=1}^{m}\left\langle\mathrm{~d} z_{p_{1}},\left(\frac{\partial}{\partial u_{i}}\right)\right\rangle^{\langle-2\rangle} \frac{\partial}{\partial z_{p_{2}}^{\langle-2\rangle}} .
\end{aligned}
$$

Substituting the vector field $\left(\partial / \partial u_{i}\right)^{\langle-1\rangle}$ from (66) into (67), we get

$$
\begin{aligned}
\left(\frac{\partial}{\partial u_{i}}\right)^{\langle-2\rangle}= & \sum_{j_{1}, j_{2}=1}^{n}\left(\frac{\partial \bar{\Phi}_{j_{2}}}{\partial x_{j_{1}}}\right)^{\langle-1\rangle}\left(\frac{\partial \bar{\Phi}_{j_{1}}}{\partial u_{i}}\right)^{\langle-2\rangle} \frac{\partial}{\partial x_{j_{2}}} \\
& +\sum_{j_{1}=1}^{n} \sum_{p_{2}=1}^{m}\left(\frac{\partial \chi_{p_{2}}}{\partial x_{j_{1}}}\right)^{\langle-1\rangle}\left(\frac{\partial \bar{\Phi}_{j_{1}}}{\partial u_{i}}\right)^{\langle-2\rangle} \frac{\partial}{\partial z_{p_{2}}^{\langle-1\rangle}}+\sum_{p_{1}=1}^{m}\left(\frac{\partial \chi_{p_{1}}}{\partial u_{i}}\right)^{\langle-2\rangle} \frac{\partial}{\partial z_{p_{1}}^{\langle-2\rangle}} .
\end{aligned}
$$

From (68) follows that

$$
\left(\frac{\partial}{\partial u_{i}}\right)^{\langle-2\rangle} \in \operatorname{span}_{\mathscr{K}}\left\{\frac{\partial}{\partial x}, \frac{\partial}{\partial z^{\langle-1\rangle}}, \frac{\partial}{\partial z^{\langle-2\rangle}}\right\}
$$

i.e. for $l=2$ (i) is satisfied. Moreover, the coefficients of $\left(\partial / \partial u_{i}\right)^{\langle-2\rangle}$ contain only the backward shifts of the partial derivatives of $\bar{\Phi}$ and $\chi$ up to order 2, i.e. depend only on the variables $x, z^{\langle-1\rangle}$ and $z^{\langle-2\rangle}$. Consequently, (ii) is also satisfied.

Continuing in the similar manner, one can prove that the lemma holds for all $l=1, \ldots, k^{\star}+1$.

\section{Lineariseeritavus olekuteisenduse abil}

\section{Tanel Mullari ja Ülle Kotta}

On esitatud tarvilikud ja piisavad tingimused diskreetaja mittelineaarse mitme sisendiga dünaamilise süsteemi lineariseeritavuseks olekuteisenduste abil. Tingimused on kirjeldatud süsteemi dünaamika poolt defineeritud vektorväljade tagasinihete abil. Samuti on näidatud, kuidas leida olekuteisendust juhul, kui tingimused on rahuldatud. Artiklis toodud tingimusi on võrreldud varasemalt sama aparatuuri abil leitud tingimustega, kus lisaks olekuteisendusele on lubatud ka staatiline olekutagasiside: on näidatud, millised tingimused lisanduvad, kui olekutagasisidet ei saa kasutada. Teoreetilisi tulemusi on illustreeritud näitega. 